\title{
The Energy Levels of Oligothiophenes in the
}

\section{Higher Excited Triplet States}

Yosuke Oseki, Mamoru Fujitsuka, Masanori Sakamoto, Xichen Cai, and Tetsuro Majima*

The Institute of Scientific and Industrial Research (SANKEN), Osaka University, Mihogaoka 8-1, Ibaraki, Osaka 567- 0047, Japan

\section{Contents}

1. ns-ns 2-LFP of 4T in THF in the presence of 1-methylnaphthalene (Figure S1).

2. ns-ns 2-LFP of 3T in THF in the presence of tetramethylpyrazine and 4-methylanisole (Figure S2).

3. ns-ns 2-LFP of $5 \mathrm{~T}$ in THF in the presence of aniline and 1,4-dimethoxybenezene (Figure S3).

4. ns-ns 2-LFP of 2T in THF in the absence of the energy quencher (Figure S4). 


\section{1. ns-ns 2-LFP of 4T in THF in the presence of 1-methylnaphthalene.}

Figure S1 shows the time profiles of $4 \mathrm{~T}$ in THF in the presence of 1-methylnaphthalene $(1.0 \mathrm{M})$ as $\mathrm{Q}$ during the ns-ns 2-LFP. This absorption can be assigned to $4 \mathrm{~T}\left(\mathrm{~T}_{1}\right)$ generated by the first 355-nm nslaser flash. The bleaching of the absorption of $4 \mathrm{~T}\left(\mathrm{~T}_{1}\right)$ was observed with the second 532-nm ns-laser irradiation at $1 \mu$ s after the first laser, indicating the TET from $4 \mathrm{~T}\left(\mathrm{~T}_{n}\right)$ to aniline. The bleaching of the absorption of $4 \mathrm{~T}\left(\mathrm{~T}_{1}\right)$ was almost recovered at $3.5 \mu \mathrm{s}$, indicating the diffusion-controlled back TET from 1-methlnaphthalene $\left(\mathrm{T}_{1}\right)$ to $4 \mathrm{~T}$.

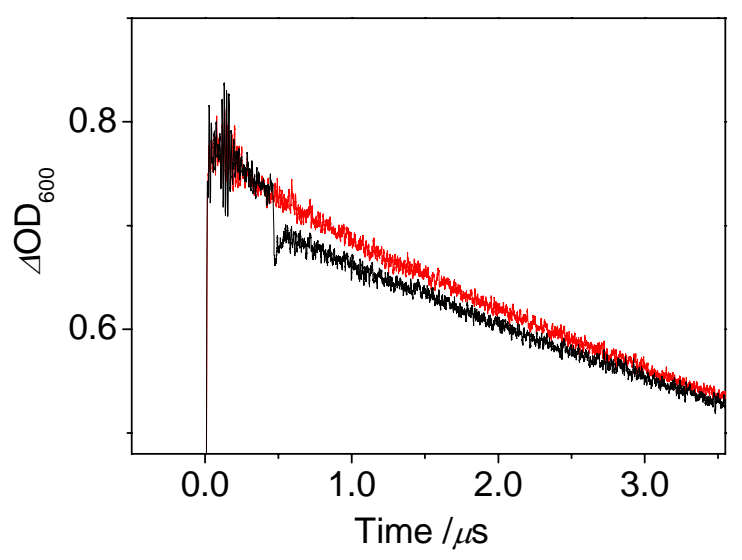

Figure S1. The time profiles of $\triangle \mathrm{OD}$ at $600 \mathrm{~nm}$ of $4 \mathrm{~T}$ in THF in the presence of 1-methylnaphthalene (1.0 M) during the ns-ns two-color two-laser flash photolysis (first laser: $355 \mathrm{~nm}$, fwhm $5 \mathrm{~ns}, 5 \mathrm{~mJ}$ pulse $^{-1}$, second laser: $532 \mathrm{~nm}$, fwhm $5 \mathrm{~ns}, 20 \mathrm{~mJ}$ pulse $^{-1}$ ). The second laser was irradiated at $0.5 \mu$ s after the first laser. Red and black lines show the one- and two-laser photolysis, respectively. The spike signal at the second laser flash is due to the laser scattering. 


\section{2. ns-ns 2-LFP of $3 T$ in THF in the presence of tetramethylpyrazine and 4-methylanisole.}

Figure S2(A) shows the time profiles of 3T in THF in the presence of tetramethylpyrazine $(1.0 \mathrm{M})$ as Q during the ns-ns 2-LFP. This absorption can be assigned to 3T( $\left.\mathrm{T}_{1}\right)$ generated by the first 355-nm nslaser flash. The bleaching of the absorption of 3T( $\left.T_{1}\right)$ was observed with the second 532-nm ns-laser irradiation at $1 \mu \mathrm{s}$ after the first laser, indicating the TET from $3 \mathrm{~T}\left(\mathrm{~T}_{n}\right)$ to tetramethylpyrazine. Similar bleaching was confirmed in the presence of 1,4-dimethoxybenzene (1.0 M) (Data not shown). On the other hand, the bleaching of the absorption of $3 \mathrm{~T}\left(\mathrm{~T}_{1}\right)$ was clearly weaken in the presence of 1methylanisole (1.0 M) (Figure S3(B)), indicating that $5 \mathrm{~T}\left(\mathrm{~T}_{n}\right)$ is close to 4-methylanisole $\left(\mathrm{T}_{1}\right)$. Therefore, the $E\left(\mathrm{~T}_{n}\right)$ of $3 \mathrm{~T}$ was estimated to be $3.4 \pm 0.1 \mathrm{eV}$.

(A)

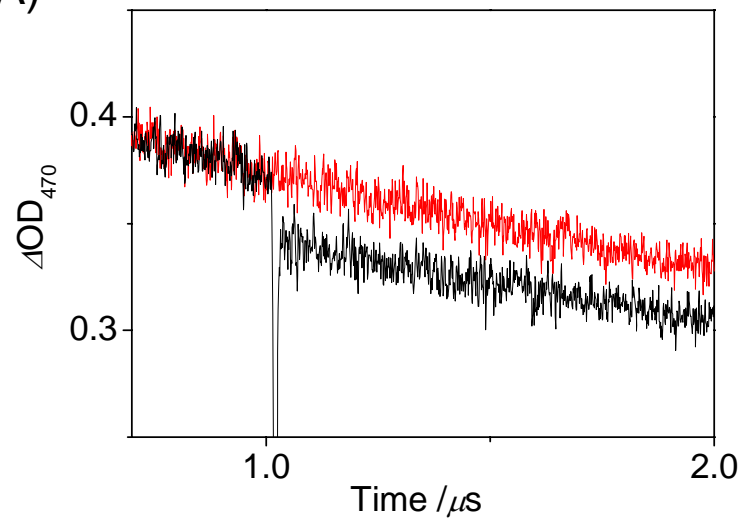

(B)

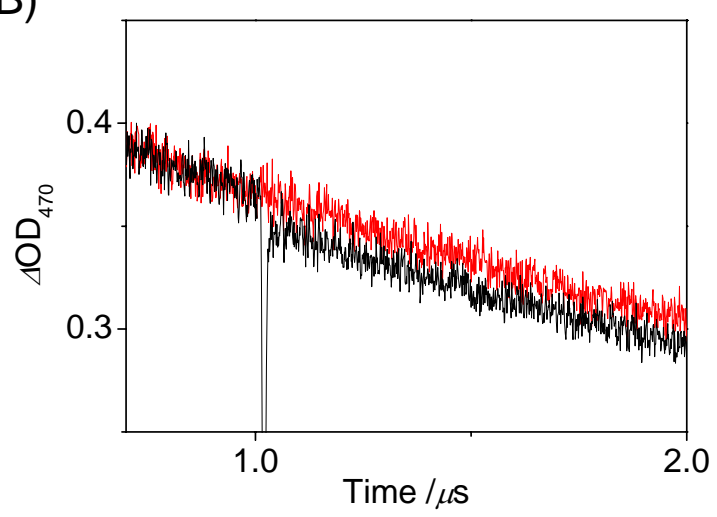

Figure S2. The time profiles of $\Delta \mathrm{OD}$ at $470 \mathrm{~nm}$ of $3 \mathrm{~T}$ in THF in the presence of tetramethylpyrazine (A) and 4-methylanisole (B) (1.0 M) during the ns-ns two-color two-laser flash photolysis (first laser: $355 \mathrm{~nm}$, fwhm $5 \mathrm{~ns}, 3 \mathrm{~mJ}$ pulse ${ }^{-1}$, second laser: $532 \mathrm{~nm}$, fwhm $5 \mathrm{~ns}, 10 \mathrm{~mJ}$ pulse ${ }^{-1}$ ). The second laser was irradiated at $1 \mu \mathrm{s}$ after the first laser. Red and black lines show the one- and two-laser photolysis, respectively. The spike signal at the second laser flash is due to the laser scattering. 


\section{3. ns-ns 2-LFP of $5 T$ in THF in the presence of aniline and 1,4-dimethoxybenezene.}

Figure S3(A) shows the time profiles of $5 \mathrm{~T}$ in THF in the presence of aniline (2.0 M) as Q during the ns-ns 2-LFP. This absorption can be assigned to $5 \mathrm{~T}\left(\mathrm{~T}_{1}\right)$ generated by the first 355-nm ns-laser flash. The bleaching of the absorption of $5 \mathrm{~T}\left(\mathrm{~T}_{1}\right)$ was observed with the second 532-nm ns-laser irradiation at 1 $\mu$ s after the first laser, indicating the TET from $4 \mathrm{~T}\left(\mathrm{~T}_{n}\right)$ to aniline. Similar bleaching was confirmed in the presence of benzo[b]thiophene $(2.0 \mathrm{M})$ (Data not shown). On the other hand, almost no bleaching was confirmed in the presence of 1,4-dimethoxybenzene (2.0 M) (Figure S3(B)), indicating that 5T( $\mathrm{T}_{n}$ ) lies between 3.08 and $3.25 \mathrm{eV}$, i.e., $3.2 \pm 0.1 \mathrm{eV}$.

(A)

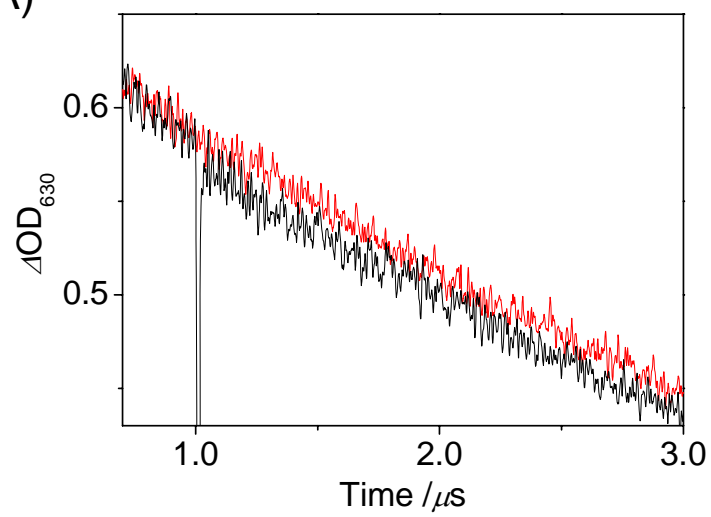

(B)

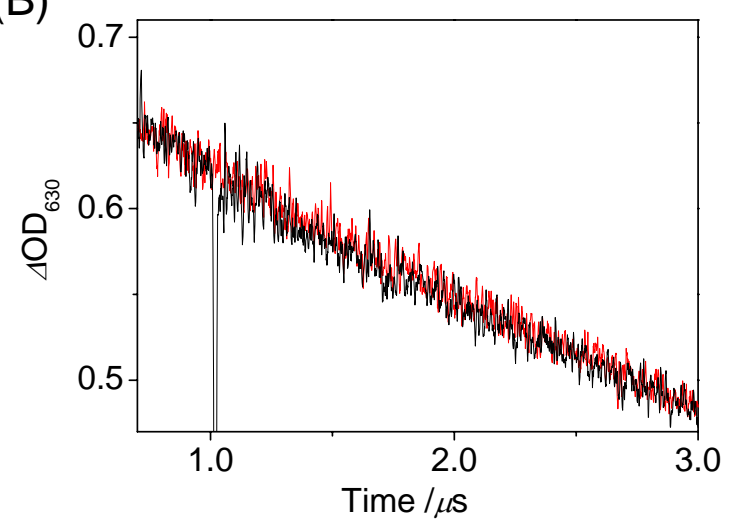

Figure S3. The time profiles of $\Delta \mathrm{OD}$ at $630 \mathrm{~nm}$ of $5 \mathrm{~T}$ in THF in the presence of aniline (A) and 1,4dimethoxybenzene (B) (2.0 M) during the ns-ns two-color two-laser flash photolysis (first laser: 355 nm, fwhm 5 ns, 1.5 mJ pulse ${ }^{-1}$, second laser: $532 \mathrm{~nm}$, fwhm $5 \mathrm{~ns}, 20 \mathrm{~mJ}$ pulse $^{-1}$ ). The second laser was irradiated at $1 \mu \mathrm{s}$ after the first laser. Red and black lines show the one- and two-laser photolysis, respectively. The spike signal at the second laser flash is due to the laser scattering. 


\section{4. ns-ns 2-LFP of $2 \mathrm{~T}$ in THF in the absence of the energy quencher.}

Figure S4 shows the time profiles of 2T in THF during the ns-ns 2-LFP. This absorption can be assigned to $2 \mathrm{~T}\left(\mathrm{~T}_{1}\right)$ generated by the first 308-nm ns-laser flash. The bleaching of the absorption of 2T( $\left.\mathrm{T}_{1}\right)$ was observed with the second 355-nm ns-laser irradiation at $1 \mu$ s after the first laser. Therefore, it was difficult to estimate the $E\left(\mathrm{~T}_{\mathrm{n}}\right)$ of $2 \mathrm{~T}$ using the ns-ns 2-LFP.

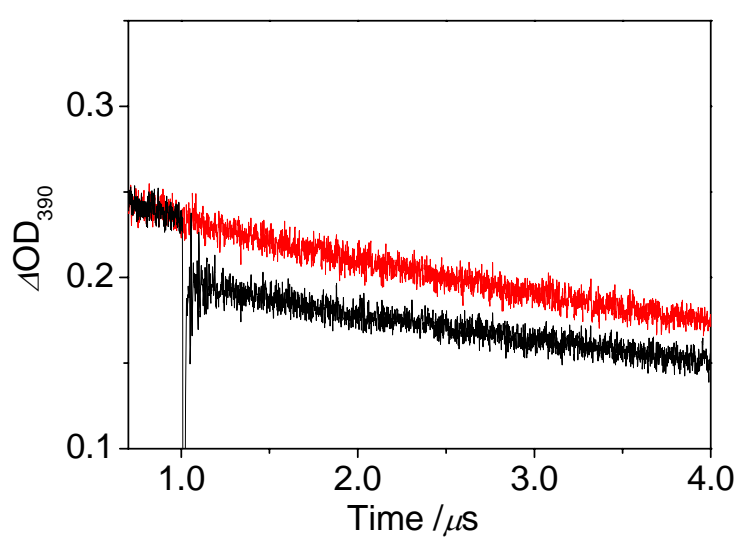

Figure S4. The time profiles of $\Delta \mathrm{OD}$ at $390 \mathrm{~nm}$ of $2 \mathrm{~T}$ in THF during the ns-ns two-color two-laser flash photolysis (first laser: 308 nm, fwhm 40 ns, 5 mJ pulse ${ }^{-1}$, second laser: 355 nm, fwhm 5 ns, 10 mJ pulse${ }^{1}$ ). The second laser was irradiated at $1 \mu$ s after the first laser. Red and black lines show the one- and two-laser photolysis, respectively. The spike signal at the second laser flash is due to the laser scattering. 